\title{
Verhandlungen über die Hausweberei im Kloster der Tertiarier zu Köln.
}

\author{
Von
}

\section{Leonard Korth.}

Am 20. November 1371 war in Köln der Aufstand des Wollenamtes und seiner Genossen blutig niedergeworfen worden 1. Die wieder zur Herrschaft gelangten Geschlechter erblickten eines der wirksamsten Mittel, um für alle Folgezeit den Regungen gewaltthätigen Uebermuthes unter den Zlunften vorzubeugen, in einer weitgehenden gesetzlichen Einschränkung des Gewerbebetriebes. Mag in älteren Schilderungen die vormalige Zahl der Weber in der rheinischen Hauptstadt auch noch so stark abertrieben worden sein 2: erwägt man die hervorragende Bedeutung, welche dic kölnische Waare auf den Tuchmärkten des Mittelalters unleugbar besass, so muss es als eine ganz erhebliche Herabminderung gelten, wenn das Eidbuch rom Jahre 1372 fur die gesammte Stadt fortan nur zweihundert Webstuhle zulassen will ${ }^{3}$, und die Ueberlieferung, dass die Textilindustrie im bergisehen Lande und am Niederrhein

1) Die weverslaicht, Chroniken der deutschen Städte Bd. 12 (Cöln 1), S. $243 \mathrm{ff}$; Dat nuwe boich, daselbst S. $275 \mathrm{ff}$.; $80 \mathrm{~g}$. Koelhoff'sche Chronik von 1499, daselbst Bd. 14, S. 706 ff.; Limburger Chronik, Mon. Germ., Deutsche Chroniken Bd. 4I, S. 61; vgl. Ennen, Geschichte der Stadt Köln Bd. 2, S. $667 \mathrm{ff}$.

2) Ennen a. a. O. S. 681; G. Schmoller, Die Strassburger Tucherund Weberzunft (Strassburg 1879), S. $434 \mathrm{f}$; K. Hegel, Städte und Gilden der germanischen Völker im Mittelalter Bd. 2 (Leipzig 1891), S. 353.

3) Es wird bestimmt, dat man neit me halden nog haven in soele nu nog nummerme mit eingin sachen sunder argelist dan zweihundert weivergezauwin, ind dat dat unse heirren selver bewarin solen ind doen bewarin. dat der neit me inwerde zo ewigin dagin', W. Stein, Akten zur Geschichte der Verfassung und Verwaltung der Stadt Köln im 14. und 15. Jahrhundert Bd. 1 (Bonn 1893) S. $89 \S 11$. 
den Scbaaren der damals aus Köln ausgetriebenen Weber ihren Ursprung verdanke, ist keineswegs ganz unglaubwürdig. So kurzsichtig hatte allerdings der Hass die Herren vom Stadtregiment nicht gemacht, dass sie einen der wichtigsten Gewerbszweige geradezu abgescbnitten hätten. Nachdem sie zehn Jahre lang die Gemeinde ihre Macht hatten füblen lassen, gewäbrten sie aus freien Stucken den Webern die Erlaubniss zur Bescbäftigung von weiteren hundert Stülılen ${ }^{1}$.

Der Grundsat\% einer Betriebsbeschränkung wurde auch nach der Unwälzung vow Jahre 1396 nicht aufgegeben, allein der demokratische Rath brachte ihn in anderer Form und vor allem in anderer Absicht zur Geltung. Politische Bedenken konnten fur ihn nicht mehr bestimmend sein, nachdem der Verbundbrief alle (ienossenschaften zu einer festen Einung zusammengeschlossen hatte. Aus dem Wortlaute der Verordnungen geht denn auch klar hervor, dass es sich bloss um polizeiliche Massnahmen, um die technische Beaufsichtigung des Gewerbes und nicht zuletzt um die Besteuerung des Betriebes bandelt, an eine Beengung der Gesammteutwicklung aber ist nicht gedacht.

So trifft der Amtsbrief der Leinenweber vom Jahre 1397 die Bestimmung, dass jeder Meister, der um zwei Gulden die Mityliedschaft erworben habe, nur einen einzigen Webstuhl besitzen durfe, fugt dann aber hinzu: ,ind so wilch me getzauwen ${ }^{2}$ dryven ind halden wilt, die sall gheven vur eyne eickliche me getzauwe 6 marck'; und ferner: so wilch meister, die dem ampte dry jaire gedient hait ind myt $v$ e i $r$ getzouwen sitzen wulde, die sal van der veyrder getzouwen eyn vol harnesch baven ind halden in oirber der stede ${ }^{\prime}{ }^{3}$. Ausgeschlossen war allerdings eine solche Erweiterung des Betriebes dem Anscheine nach für das Wollenamt: ,Vort so ensall geyn man, meister noch broider, me wirken dan mit tzwen getzauwen up syn anpte ${ }^{6}$.

1) ,Van den wevergetzaüwen is oeverdragen, dat man boyven drahundert nyet baven noch entfangen en suele bynnen desen 10 jairen dat dis oeverdrach weyren sal' etc., Eidbuch rom Jahre 1382, W. Stein, a. a. 0. Iid. 1, S. $126 \S 9$.

2) Der Seltsamkeit wegen merke ich bier an, dass neuerdings, getzauwe' für „ein besonders kostbares Tuch“ gehalten worden ist und zwar von A. Henning, Steuergeschichte von Köln . bis zum Jahre 1370 (Leipziger Diss., Dessau 1891) S. 68.

3) Quellen zur Gesch. der Stadt Köln Bd. 6. S. 527.

4) a. a. O. S. 570; vgl. Schmoller, Tucher. und Weberzunft S. 453. 
Der Rath hatte bei der Durehführung dieser Verordnungen sein Augenmerk alsbald auch auf diejenigen Klöster der Stadt gerichtet, in denen die Handarbeit allmählich in die Formen eines eigentlichen Gewerbebetriebes ibergegangen war. Allein dass man die in den Ordenshäusern thätigen Handwerker den Verbundbrief beschwören liess ${ }^{1}$, genügte nicht, um allen weiteren Schwierigkeiten vorzubeugen.

- Von vornherein muss freilich bemerkt werden, dass die Verwicklungen, die sich noch ergaben, recht harmlos erscheinen, wenn man sie etwa mit den langwierigen Kämpfen der Bürgerschaft gegen den Weinzapf der Geistlichkeit in Vergleich zielıt ?. Der airgerlichen Gewinnsucht der reichen Stifter, welche die Unverletzlichkeit ihrer Inımunitätsbezirke zur Errichtung abgabenfreier Weinsehänken missbrauchten, stehen hier bessere Gesinnungen gegentuler. In einer Reihe von geistlichen Genossenschaften war der alte Satz der Benediktiner-Regel [c. 48] nie in Vergessenheit gerathen: Tunc vere monachi sunt, si labore manuum suarum vivunt ${ }^{3}$ und eben zu A usgang des vierzehnten Jahrhunderts hatten ihn die Brijler vom gemeinsamen Leben auf's neve zu Ehren gebracht. Aber auch die ernste Sorge um den täglichen Unterbalt trieb die Mitglieder der besit\%losen Vereinigungen zu emsiger Thätigkeit an. Förderlich war ihnen dabei wiederum das Wohlwollen des Volkes, das von jeher der freiwilligen Armuth seine besondere Theilnahme \%ugewandt batte, mit Vorliebe aber gerade die Nachfolger des beiligen Franziskus unter seinen Scbutz stellte. Wir haben es durchaus nicht mit einem vereinzelten Falle $z \|$ thun, wenn im Beginne des funfzehnten Jabrhunderts in dem kleinen Stätchen Goch die Bügerschaft zusammen mit ihrem Pfarrer und selbst mit dem herzoglichen Amtmanne dafür eintritt, dass den Tertiarierinnen in dem Grossen Konvente die Erlaubniss zur Leinwandweberei nicht entzogen werde ${ }^{4}$.

Je leichter unter solchen Umständen die Klosterinsassen ihren Abnehmerkreis zu erweitern im stande waren, um so begreiflicher finden wir es, wenn nun die Zünfte ihrerseits einer iibermässigen

1) Vgl. unten die Zeugenaussagen in der Urkunde rom 8 . Februar 1400.

2) Ennen, Geschichte der Stadt Köln Bd. 2, S. $758 \mathrm{ff}$.

3) Ueber Handarbeit in den Kilöstern vgl. auch H. Denifle im Archir für Litteratur- und Kirchengeschichte des Mittelalters Bd. 1, S. 180.

4) Vgl. den immer noch werthvollen Aufsatz von P. B. Bergrath, Das Wüllenamt zu Goch, Annalen d. Hist. Ver. f. d. Niederrhein Heft 5, S. 130 f.; dazu die Urkunde vom 30. Januar 1422, daselbst Heft f; S. 71 f. 
Ausdebunng der Gewerbthätigkeit in den geistlichen Niederlassungen zu steuern suchten.

Auf Bemübungen solcher Art beziehen sich die drei Urkunden, die im folgenden zum Abdrucke gebracht werden. Sie vergegenwärtigen uns Verhandlungen des Rathes mit den Bridern rom dritten Orden des heiligen Franziskus in Kloster ad Olivas, das hier unter seinem volkstüulichen Namen, zu den Olvonden' begegnet ${ }^{1}$. Einer Erläuterung sind die Aktenstücke nach den, 'was oben bemerkt worden ist, nicht bediurftig. Hervorzuheben ist nur aus den Zengenaussagen vom 8 . Februar 1400, dass unter den für den Konvent beschäftigten Webern nicht mehr als zwei Klosterbrider [IV.IX] sich befinden. Sodann verdient Beachtung, dass nach der Niederlage des Wollenamts eine Vermehrung der Webstiihle in Kloster von 25 auf 32 durch die Einfubrung der, gisselsgezauwe' stattgefundeu bat. Bemerkenswerth darf endlich noch die örtliche Vereinigung der Leinen- und der Wollenweberei erscheinen. Von den neun zan Verbör gelangenden Zeugen sind zwei Leinenweber [I. VIII], drei Wollenweber [II. III. V.], drei Spuler [VI. VII. IX.], wäbrend Bruder A mold ron Koblenz [IV.] seine Spezialität nicht bezeichnet. Die Reform, welche der Rathsbeschluss vom 22. Oktober 1400 durchfuhrt, setzt nicht nur die Gesammtzahl der Webstuhle von zweiunddreissig auf zwölf herab, sondern bringt auch durch die völlige Beseitigung der Wollenweberei und der ,gisselsgezauwe' den Grundsatz einer strengen Arbeitstheilung zum Ausdruck ${ }^{2}$. In einem grösseren Zusammenhange betrachtet stellen diese unscheinbaren Vorgänge immerhin eine Stufe in der Ausbildung des Zunftrechtes dar, dessen Anfänge ja nicht etwa in ferner Vorzeit zu suchen sind, das vielmehr, nach G. Schmoller's treffender Bemerkung, gerade gegen das Jahr 1400 in sein Mannesalter eintritt und nun sich in seinen Grundgedanken erst vollends entwickelt ${ }^{3}$.

Es eriibrigt mir noch, meinen Freunden Herrn Pfarrverwalter Karl Füss enich in Morken und Kaspar Keller in Köln, jenem

1) Näheres bei Aeg. Gelenius, De admiranda magnitudine Coloniae S. 519 f.; Ennen, Geschichte der Stadt Köln Bd. 3. S. 999.

2) Ueber die Arbeitstheilung, insbesondere zwischen Leinen- und Wollweberei vgl. Tr. Geering, Handel und Industrie der Stadt Basel (Basel 1986) S. 249.

3) Schmoller, Tucher- und Weburzunft S. 471. 
für die Mittheilung der Urkunde vom 8. Februar 1400 (I), diesem für die Abschriften aus den Kölner Rathsprotokollen (11. III.), meinen herzlichen Dank zu sagen.

I.

1400 Februar 8, Köln. - Zeugenaussagen über den Betrieb der Weberei im Franziskanerkloster zu den Olvonden in Köln seit den Zeiten der Weberschlacht.

In goitz namen amen. Kunt si allen luden de dit intgaynwordige offenbair instrument an seynt of | borent lesen, dat want, asman sait, tusschen den geistlichgen broderen ind religiosen personen deym/meister ind den broederen des gemeynen conventz van deym dirden orden ind regulen sent Franciscus zo den Olronden in Coelne, de man beist de broder der penitencien, van eyure sijden ind van den irberen luden den lijnenweferen ind vort van anderen ampten in Coelne van der ander sijden uperstanden was ind is zwist ind zweyunge ind de eczwat lange in kijfe ind in sweyongen under eynander gestanden hait as umb gezauwen willen de de vurschreven meister ind brodere lange zijt ind jair in irme convent vry los unverboden ind sunder emans messen of besien vur des wullenamptz slacht gerast ind geroit besessen batten; ind her enboven baynt de lijnenwefer den vurscbreven meister ind broederen des conventz doyn gebeden ind heisschen vur den vollen rait unsser heirren van Coelne ind willend in ur vryheyt de si gehat ind besessen haynt, as vurschreven steit, doyn benemen. Ind umb dat unsse heirren alle sachen, we man dat van alders zo balden plach ind gebalden ward kủndijch sy, so baint de vurschreven broeder irber lude in ir vurscbreven convent laissen komen de mit namen berna geschreven stient ind de kunsschaff as vurscbreven steyt gesacht haynt by deym eyde den si unssen herren vamme rade ind der stede van Coelne ind vort der gantzer gemeynden in deym verbundbrefe gedain haint, ind de broeder by deym eyde den si deym orden gedain haynt, ind were is noit, si woulden it ouch lijflichen zo den beilgen sweren so wat si gesacht betten, dat it also were. Ind herumb so is in intgaynwordicheyt min notarius underschreven ind gezuchen de bey unden geschreven stient ind herzo geheyssen ind gerofen synt erschenen is in deym irsten: [I.] Clais Erfort de alt was, as hey sachte, 80 jair in me, de sachte, dat is were me dan 30 jair vur der slacht des wullenamptz dat hey plach den broderen in deym 
convent zo den Olvonden vurschreven zo wefen up den lijnengezauwen, do hatten de broeder in irme convent da man up zo wefen plach 9 lijnengezauwen, 4 wullengezauwen, swa zartzengezauwen, ein stoillackgezauwe, 9 tirteysgezauwen, ind dat man in den 30 jaren nyet zo messen en plach noch geyne maisse en was, ind were des noit, dat weulde hey lijflijch zo den heilgen sweren dat it also were as vurgeschreven steit. [II.] Item Rychel Koch de alt was, as hey sachte, 70 jair ind me, de $\mathrm{e}^{\mathrm{a}}$ saichte dat is wail were 40 jair dat he wefede up den wullengezauwen in dey'm convent ind dat do waren in deym convent da man uppe zo wefen plach 9 lijnengezauwen, 8 gisselezgezauwen, 4 wullengezauwen, 8 tyrteysgezauwen, 2 zarczengezauwen, 1 stoillackgezauwe, ind dat man do nyet zo messen en plach noch geyn maisse en was ind dat hey alle sijne dage nye maisse in deym convent in sege, dat nam bey up den eyt den hey unssen herren ramme rade, der stede van Coelne ind vort der ganczer gemeynden up deym verbundbreif gedain Lait etc. etc. [wie oben]. [III.] Item Jacob Koch de alt was, as hey sachte, 68 jair ind me, de sprach dat is wail were 40 jair nu hey wefede up den wullengezauwen in deym convent ind do waren in deym convent etc. etc. [wie oben]. [IV.] Item broder Arnolt vau Kovelens eyn broder zo den Olvonden vurschreven, de ${ }^{b}$ alt. was, as hey sachte, 65 jair ind me, dat is wail were 37 jair dat bey in dat convent vurschreven quam ind den orden entfynge dat do waren 9 lynengezauwen, 4 wullengezauwen, 2 zarczengezau wen, eyn stoillaegezauwe, 8 tirteysgezauwen ind 8 gisselczgezauwen da man up zo wefen plach in deym convent ind nam dat up den eyt den bey deym orden gedayu bait ind were is noit, bey woulde it lijflijeb zo den heilgen sweren dat it also sy as vurschreven steyt. [V.] Item Geirlach Veirpennynck de alt was, as hey saclite, 60 jair ind me, de sprach, dat is wail were 36 of 37 jair dat hey $z o$ wefen plach up den wullengezauwen in dem convent, dat do waren in deym convent 4 wullengezauwen etc. etc. [wie oben; Eid auf den Verbundbrief]. [VI.] Item Gerard Erenberch de alt was volna, as bey sachte, 60 jair, de spraich dat hey spoilde in deym vurschreven convent in der Romervard ${ }^{1}$ ind dat do waren in deym convent 9 lijnengezauwen, 4 wullengezauwen, 2 zarzengezauwen, eyn stoillackgezauwe, 9 tirteysgezauwe ind 6 of 7 gisselczgezauwen

a) de. b) dat.

1) Es ist wohl der Römerzug Karls IV. vom Jahre $135 \overline{\text { gemeint. }}$ 
da man up worte ind zo wefen plach etc. etc. [wie oben: liein Mass; Eid auf den Verbundbrief']. [VII.] Item Tiel Zornnynck, de alt was 56 jair as hey sachte. de sprach dat hey spoilde in deyn convent in der Romervart ind dat do waren in deym convent 9 lijnengezauwen etc. etc. [uie oben $\left.u^{\circ} V I\right]$. [VIII.] Item Johan van Roydingen, de alt was as hey sachte 50 jair of me. de sprach dat des wail were 29 jair dat hey up den lijnengezauren in deym convent zo wefen plach, dat do waren in deym convent 9 lynengezauwen da man up zo wefen plach ind dat man aldo nyet zo messen en placb etc. etc. [Kid auf drn Verbundbriet']. IX. Item broeder Francke der meister zo den Olvonden, de sachte dat hey were alt 46 jair ind me, ind dat is wail were 36 jair, dat hey spoilde in dem convent ${ }^{1}$, dat do waren in deym convent do man up zo wefen plach 9 lijnengezauwen, 4 wullengezauwen, 2 zarczengezauwen, eyn stoillackgezauwe, 8 tirteysgezauwen ind 8 gisselc\%gerauwen ind nam dat up den eyt den hey dem orden vurschreven gedain hatte, ind were is noit, hey weulde it lijflijch zo den heilgen sweren dat it also si as vurschreven steit; ind ouch is eme kun. dijch, dat do geyn nuaisse en was noch zo messen en plach.

Ind want ich over allen vurschreven sachen gewiest byn ind de gesien ind gehoirt hain as vurschreven steit, so baint de vurschreven meister ind broder des conventz zo den Olvouden vurschreven gesunnen van mir gemeynen notarius underschreven, dat ich in in duytzen eyn instrument of viel dichten ind schrijfen wille in der bester formen dat dat sijn mach. Dit is geschiet in den jairen uns herren do man schreyf dusent veirhundert in der eychter indiccien ind imme eichten dage in der spurckelen tusschen nonen ind vesperen; da synt over ind an gewiest de irber bescheydine lude Nolde van Lieber, Wilhelm van Reyde ind Jacob Wolf gezuyge de herzo gebeden ind gerofen synt zo eyme gezuychnisse alre dynge vurschreven.

[Notariatszeichen]. Et ego Gerardus Henrici de Nussia clericus Coloniensis publicus imperiali auctoritate curieque Coloniensis notarius iuratus premissis omnibus et singulis etc. presens (inter)fui etc. ideo hoc instrumentum exinde confeci quod manu mea propria scripsi etc.

1) Der zehnjährige h́nabe wurde sonach schon als Spuler beschäftigt. 


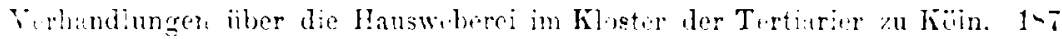

Auj der Rücliseite ron etua gleichzeitiger Hand, stark verblasst: Iustrumentum in theutonico, quod olim fratres non stringebantur ad certum numerum instrumentornm textrinorum.

Nota. Domini communitatis artaverunt fratres post suppressionem consulum et scabinorum ad instanciam et voluntatem officiurum al habendum duntaxat 6 lijnenghetou, 2 saertssengheton, 1 stoillakens, 4 tyrteysgbetou libere sine omni gravamine preterquam quod magistri officiorum explorare possint et debeant an habeant [iustam men]suram ....

Darunter ton späterer Hand 15. Jhdts.: Dit is der breif van den weyvegeczauwen. Aufschriften 17. Jhdts.: Vertrag zwischen den wullen- und leinenweberen und den broderen ad Olivas. Littera E. concernit conventum ad Olivas Coloniensem.

Original auf Pergament, z. Z. im Besitze des Herrn Pfarrverwalters $K$. Fïssenich zu Morken.

II.

1400 Juli 16. - Der Rath der Stadt Köln gestattet den Briidern in Kloster zu den Olvonden, sechs Webstihle zu beschäftigen, falls sie der Aufsicht [des Leinenamtes] sich unterwerfen wollen.

Van den Olvonden.

Id sij zo wissen, dat unse berren vamme raide haint oeverdragen van deu Olvonden bynnen Coelne, also dat man yr werk besein und meszen sall as man vur 40 jaren zo besein ind zo meszen plach. Ind wert sache, dat die Olvonde yr werk nyet enwolden laissen besein noch meszen, so verbeident unse herren vesticlichen, dat geyne yrre burgere, burgerssen off yngesessen den Olvonden eynich werk zo wyrken zubringen soelle; ind ouch dat geyne burger, burgersse off yngesessen den Olvonden yn dem ampte, dat sij hantieren plegen, deynen soelen. Ouch baint unse herren den Olvonden georloft, asverre as sij guetliche lijden $\mathrm{yr}$ werk zo besein ind zo messen as vurscreven steit, dat sij bynnen yrre wonyncgen 6 getzouwen haven ind halden moegen. Ind were sache, dat sij dar boven me getzouwen begerden zo hain, die suellen sij wynnen an genaden unser herren vamme raide. Datum et concordatum anno domini milesimo quadringentesimo crastino festi divisionis apostolorum. Stadtarchiv Köln, Rathsprotokolle Bd. 1, Bl. 16. 
III.

1400 Oktober 22. - Der Rath der Stadt Köln bestimmt die Zahl der Webstühle im Kloster zu den Olvonden, unterstellt die Gewebe der Aufsicht des Leinenamtes und rerpflichtet die Brüder zu den gewöhnlichen Abgaben.

\section{Van den Olvonden.}

Also as dye van dem lynenampte lanege zijt her myt den Olvonden bynnen Coelne zwistich under en ander syn geweist, so haint unse herren vam raide da van overdragen ind yre vrunde, myt namen herren Johan Florijn ind Peter van Wichterich da bij geschickt, umb eyndracht ind vruntschaff tusschen den van dem lynenampte ind den Olvonden zo maichen, na dem unse herren davan overdragen hain na formen ind manyren hijr na gesehreven: Also dat dye Olvonde bynnen yrme huse ind woningen bynnen Coelne ind yre nakomelinge baven ind halden moegren, ungewunuen tuain yemande, 6 lijnengetzouwen ind nyet me, 4 tirteyssgetzouwen ind nyet me, 1 scbarzengetzowen ind nyet me, 1 stoillakensgetzouwe ind nyet me ind eyne groisse scbarzengetzouwe; ja also, so wannee dye Olvondeu myt der groissen scharzengezouwen wirkent off wirkeu doint, dat asdan dye kleyne scharzengezouve ledich stain ind nyet gain sal, bis so lange, dat dye groisse gezouwe van dem wcrke geledicht wirt, also dat dye kleyne scharzengezouwe myt der groissen gezouwen zo eyner zijt zosamen nyet gain sullen. Ouch baint unse berren overdragen, dat dye meistere van dem ampte dye gesat synt ind gesat werden, der Olvonde werk guetliche besein ind messen sullen gelych dye ander werk plegen zo besein ind \%o messen, dat ouch dye Olvonde guetliche duegen sullen. Ind were saiche, dat dye meistere under den Olvonden eynich werk boisswerdich vunden na gesetze des ampt, so suelen dye Olvonde van alsuelchem boiszwerdigen werke yre boiszen gelden ind geven gelijch anderen van gelijchen werke greldent. Ouch sullen dye Olvonde vau yren werke dat zijsbur ys jre assijse geven gelijch anderen burgeren van dem ampte geburt zo geven. Concordatum anno $\mathrm{M}^{\circ}$ ccec $^{\circ}$ crastino undecim militum virginum.

Stadtarchiv Köln, Rathsprotokolle Bd. 1, Bl. 18. 